REVISTA MAD, Nº 35 (2016), PP. 60-74

\title{
De von Bertalanffy a Luhmann: Deconstrucción del concepto "agroecosistema" a través de las generaciones sistémicas
}

\author{
From von Bertalanffy to Luhmann: Deconstruction of the Concept "Agroecosystem" \\ through Systemic Generations
}

Lorena Casanova, Juan Martínez, Silvia López, Gustavo López

Colegio de Postgraduados, Campus Veracruz, Programa Agroecosistemas Tropicales, México.

RESUMEN

El pensamiento sistémico contribuyó al origen del pensamiento agroecológico con aportaciones desde la teoría general de sistemas y la cibernética como su base teórica primigenia. Así, la agroecología en los años 70 del siglo pasado pudo erigirse como una nueva forma de pensar y hacer ciencia en relación con el quehacer agrícola al intentar ir más allá del enfoque reduccionista predominante. El estudio de la realidad agrícola contemporánea exige planteamientos nuevos que permitan comprender sus dimensiones, para lo cual los principios basados en el modelo input-output que funciona bajo un controlador cibernético resultan insuficientes. En consecuencia, el propósito de este manuscrito fue analizar la evolución del pensamiento sistémico y su trascendencia en el pensamiento agroecológico, al brindar a éste último herramientas teórico-conceptuales para estudiar fenómenos agrícolas actuales a través de los sistemas complejos, y con ello, involucrar aspectos poco explorados en su abordaje tradicional como la historia, la política y la cultura.

PAlabras Clave: Agricultura; Agroecosistema; Pensamiento sistémico; Pensamiento complejo; Sistemas complejos

\section{ABSTRACT}

Systems thinking contributed to the origin of agroecological thought, which had as primordial theoretical basis the contributions from the general systems theory and cybernetics. Thus, agroecology in the 70s of the last century could establish itself as a new way of thinking and doing science related to agricultural activity to try to go beyond the dominant reductionist approach. However, the study of contemporary agricultural reality demands new approaches to understand it in its many dimensions. Accordingly, the purpose of this study was to analyze the evolution of systems thinking and its significance in the agro-ecological thinking by providing theoretical and conceptual tools to study current agricultural phenomena through complex systems, including unexplored aspects in its traditional approach as history, politics and culture.

KEYWORDS: Agriculture; Agroecosystems; Systemic thinking; Complex thinking; Complex systems

INTRODUCCIÓN

La actividad científica vinculada al estudio de la realidad agrícola se ha realizado fundamentalmente desde el paradigma analítico, el cual ha predominado en la ciencia desde el siglo XVII (Morin 2007). Esto es, el

\footnotetext{
REVISTA MAD - REVISTA DEL MAGÍSTER EN ANÁLISIS SISTÉMICO APLICADO A LA SOCIEDAD ISSN 0718-0527

Facultad de Ciencias Sociales, Departamento de Antropología.

Universidad de Chile

www.revistamad.uchile.cl
}

DOI: 10.5354/0718-0527.2016.42797 
predominio de un enfoque agronómico cuya prioridad es la búsqueda de la eficiencia a través de la identificación del factor limitante a lo cual subyace únicamente una racionalidad económica y tecnológica (Borsatto \& Simões do Carmo 2012). Una forma de pensar y hacer ciencia que caracterizó al modelo denominado "Revolución Verde" y que aún sigue como esencia en el discurso sobre la modernización de la agricultura en los países en desarrollo (Altieri \& Toledo 2011).

No obstante, la agricultura actual se caracteriza por la convergencia de procesos ambientales, económicos, sociales y cuya sinergia rebasa espacial y temporalmente a las unidades de producción. De ese modo, el enfoque agronómico quedó rebasado, ya que ahora también es necesario comprender el comportamiento de los mercados y las políticas agrícolas, asimismo, el contexto cultural que media dicha actividad y su antecedente histórico. Aspectos que influyen en lo que se producirá, cuándo, con qué tecnología, a qué ritmos y para qué clase de consumidores (León 2009). Un ejemplo es la agricultura mexicana contemporánea caracterizada por los efectos derivados de una crisis permanente provocada por la implementación desde hace tres décadas de un modelo de desarrollo neoliberal (Calva 2004) y por el impacto del cambio climático, ambos en una relación de exacerbación mutua (O’Brien \& Leichenko 2000). Lo anterior expresa de manera sucinta el grado de complejidad de esta agricultura y la necesidad de contar con planteamientos teórico conceptuales robustos para su comprensión, de tal forma que permitan entender el efecto de fenómenos que suceden en diferentes escalas espaciales y temporales, pero con efectos en el continuum de realidad agrícola local.

Una respuesta a lo anterior es el estudio de la agricultura desde la agroecología, una disciplina que emerge en los años 1970 del siglo pasado (Gliessman 2005; Rivera 2009). Un enfoque al que subyace el pensamiento sistémico, el cual se desarrolló a partir de aportaciones teóricas desde diferentes disciplinas emergidas en su mayoría a partir de la mitad del siglo XX (Lilienfeld 1984; Bertalanffy 1986). En los últimos años este pensamiento sistémico ha evolucionado hacia la complejidad, no así el pensamiento agroecológico, cuyos planteamientos si bien, han permitido abordar otros aspectos más allá de las interacciones ecológicas, esta evolución no ha sido suficiente para abordar dimensiones como la sociocultural y la sociopolítica. Es decir, se requiere que la agroecología construya su objeto de estudio considerando la multidimensionalidad que le es inherente, trabajando desde la inter y transdisciplina, para lo cual requiere del uso de aportaciones teóricas desde el pensamiento sistémico complejo que le permitan lograr una aproximación a la dinámica de la agricultura contemporánea. Con base en lo anterior, el presente manuscrito tiene como propósito reflexionar sobre el origen y la evolución del pensamiento sistémico, comprendiendo su influencia en el desarrollo 
del pensamiento agroecológico, esto a través de la deconstrucción ${ }^{1}$ de su unidad de estudio: el agroecosistema.

\section{EVOLUCIÓN DEL PENSAMIENTO SISTÉMICO Y SU DIFERENCIACIÓN A TRAVÉS DE LAS GENERACIONES SISTÉMICAS}

Las ideas primeras del pensamiento sistémico podrían probablemente tener su origen en la filosofía. Fernando Sáez (2009) arguye que el concepto sistema tiene referencias desde la Grecia Clásica con filósofos como Hesíodo (siglo XVII a.C.), Platón y Aristóteles (siglo IV a.C.), quienes hicieron referencia al término systêma. Sin embargo, es hasta la primera mitad del siglo XX cuando surgen una serie de ideas que se considerarán la base del pensamiento sistémico, entre las que destacan, las de Sthepen Pepper quien con su aproximación contextualista concibe al mundo como un complejo e ilimitado proceso de cambio y novedad, orden y desorden, y desde su aproximación organicista formula la categoría de "estructuras integrantes" (Lilienfeld 1984). Otras ideas fueron las expuestas por Walter Cannon (1941) en su libro La Sabiduría del Cuerpo, cuyo tema principal es el concepto de "homeóstasis" y lo planteado por Lawrence Henderson y su concepto de "equilibro", cuyo pensamiento sociológico fue basado parcialmente en analogías bioquímicas y fisiológicas (Lilienfeld 1984).

No obstante, es a partir de la segunda mitad del siglo XX cuando el pensamiento sistémico emerge como tal con la aparición de teorías nuevas e independientes: la teoría general de sistemas, la cibernética, la teoría de la información y comunicación, la investigación de operaciones, la teoría de juegos y las técnicas para simular procesos sociales y ambientales (Lilienfeld 1984). Esta efervescencia de aportaciones muestra un dinamismo tal, que difiere de la evolución del paradigma analítico, el cual ha permanecido esencialmente intacto durante más de cuatrocientos años (Morin 2007; García 2008).

Respecto a la rápida evolución del pensamiento sistémico, Jamshid Gharajedagui (2008) plantea que éste ya pasó por tres distintas generaciones de cambio en los últimos setenta años; siendo la primera aquella que trató sobre la interdependencia en el contexto de los sistemas mecánicos. Desde nuestra perspectiva, a esta generación corresponden las aportaciones de la teoría de investigación de operaciones e ingeniería de sistemas desarrolladas en Inglaterra durante la Segunda Guerra Mundial bajo el liderazgo de Edward Williams (Lilienfeld 1984). Asimismo, los primeros modelos de simulación realizados por Jay Forrester (1989)

\footnotetext{
${ }^{1}$ La teoría de la deconstrucción se considera un medio para realizar un análisis capaz de revelar dimensiones nuevas de cosas o conceptos ya conocidos (Krieger 2004; Durán 2013). Lo anterior requirió la revisión de los planteamientos de diversos teóricos del pensamiento sistémico y las principales ideas de autores abocados a la investigación agroecológica en literatura científica en motores de búsqueda y material impreso.
} 
basados en procesos de retroalimentación que él realizó para la marina de los Estados Unidos de América. Además, las primeras ideas de Ludwig von Bertalanffy (1986) en cuanto a que un sistema puede definirse matemáticamente de varias maneras.

Gharajedagui (2008) plantea que la segunda generación de pensamiento sistémico trató del doble desafío de la interdependencia y la auto-organización, en el contexto de los sistemas vivos. En esta generación podemos ubicar a Norbert Wiener y su obra Cybernetics publicada en 1948 donde plasma sus estudios sobre los sistemas recursivos al integrar la noción de feedback y la relación entre los conceptos de entropía, desorden e información (Lilienfeld 1984). Los argumentos de Bertalanffy (1986) respecto que los organismos son sistemas abiertos, capaces de permanecer en un estado uniforme, logrando evitar el aumento de entropía, e incluso lograr estados de orden y organización crecientes. Edgar Morin (2007) expresa que en las ideas de Bertalanffy hay un sistemismo fecundo que lleva en sí mismo un principio de complejidad. A la segunda generación pertenecen también las ideas de Claude Shannon y su teoría de la información, la cual en su intención original es de un alcance acotado, al referirse sólo a las condiciones técnicas que permiten la transmisión de mensajes (Lilienfeld 1984). Planteamiento fortalecido por Warren Weaver (1964), quien enfatizó las bondades de dicha propuesta y colaboró con Shannon para dar origen al modelo de comunicación de Shannon y Weaver. No obstante, es Weaver (2004) quien introduce en 1948 la palabra "complejidad" en el escenario de la ciencia con su obra denominada Science and Complexity.

A esta etapa corresponde también la aportación teórica de Jhon von Neumann plasmada en la obra On Selfreproducing Automata publicada en 1948, en la que aborda la complejidad de los "autómatas" naturales en comparación con los artificiales, así como, los trabajos de Neumann con Oskar Morgensten que dieron origen al libro Theory of Games and Economic Behaviour. Una teoría utilizada para analizar los conflictos y la competencia en la economía, pero aplicable para explicar la regulación y el control en los organismos biológicos (Lilienfeld 1984). Morin (2007) afirma que es con Neumann cuando por primera vez aparece el carácter fundamental del concepto de complejidad enlazado con los fenómenos de auto-organización.

Otros aportes teóricos en esta etapa son: Peter Checkland (1993) y los conceptos de "emergencia", "jerarquía", "comunicación" y "control" utilizados para explorar la realidad social. Idalberto Chiavenato (1997) y su teoría de sistemas para definir situaciones organizacionales $\mathrm{o}$ empresariales complejas, diagnosticar y proponer soluciones en las diferentes disciplinas especializadas de la administración. John van Gigch (1990), quien plantea que el mal funcionamiento de un sistema es causado por defectos del contenido o sustancia y asignable a causas específicas. 
También, están las aportaciones de Herbert Hart (1985), quien arguyó que la teoría de sistemas debe ser considerada la base conceptual para la investigación agrícola en el trópico, puesto que existe poca esperanza de entender los procesos agrícolas, si se siguen estudiando solamente los detalles. Un aspecto común en las ideas de los teóricos de la segunda generación, es su concepción de los sistemas como entes que pueden existir realmente al ser un agregado natural o inventado por el hombre.

La tercera generación de pensamiento sistémico según Gharajedagui (2008) responde al triple desafío de "interdependencia", "auto-organización" y "libertad de elección", en el contexto de los sistemas socioculturales. Esta generación es el resultado de la necesidad de reducir interminables complejidades y la exigencia de lograr una complejidad manejable en sistemas sociales, los cuales se caracterizan por estar abiertos, tener propósitos, ser multidimensionales, emergentes y contra-intuitivos, siendo la cultura su ADN. Es en esta etapa cuando la palabra complejidad comienza a ser protagonista, esto se expresa en las ideas de Enrique Herrscher (2005), Morin (2007) y Rolando García (2008), con sus obras Pensamiento Sistémico, Introducción al pensamiento complejo y Sistemas Complejos, respectivamente.

Es en la tercera generación que el concepto sistema es concebido como un modelo útil para el estudio de la realidad (Herrscher 2005). Esta idea coincide con lo expresado por García (2008), quien plantea que un sistema complejo es una representación de un recorte de la realidad. Por último, están los planteamientos de Niklas Luhmann con su teoría de sistemas sociales autopoiéticos, cuya arquitectura teórica-conceptual podría considerarse el inicio de una cuarta generación al plantear a los sistemas complejos como el resultado de la capacidad de éstos para producir y reproducir los elementos que les conforman y los diferencian del entorno, por ende, el término auto-organización es sustituido por el de "autopoiesis" (Luhmann 2006).

Sin embargo, esta evolución no ha sido paralela al pensamiento agroecológico, ya que, aunque la realidad agrícola contemporánea se caracteriza por su complejidad, ésta sigue siendo fundamentalmente estudiada de acuerdo con los principios sistémicos de la segunda generación, obteniendo resultados sin duda valiosos, pero parciales, lo cual impide comprenderla como una totalidad (Casanova-Pérez et al. 2015). Bajo este contexto, el pensamiento sistémico complejo que caracteriza la tercera y la cuarta generación se consideran el fundamento necesario para la consolidación de la nueva agroecología y su unidad de estudio: el agroecosistema. 


\section{DECONSTRUCCIÓN DEL CONCEPTO AGROECOSISTEMA DESDE EL} PENSAMIENTO SISTÉMICO

Las teorías son un medio para interpretar la realidad, por lo tanto, éstas no se escogen por cuan verdaderas sean, sino por lo útiles que resultan en la construcción de modelos específicos para explicar fenómenos e intentar resolver problemas en situaciones concretas (Abitbol \& Botero 2005). Esto significa que el uso de cada teoría conduce a una comprensión de la realidad agrícola relativamente distinta.

En el caso de la primera generación del pensamiento sistémico, los planteamientos de teorías como la cibernética, teoría de la investigación de operaciones y teoría de la información resultaron insuficientes para abordar la realidad agrícola, ya que sus planteamientos estaban enfocados básicamente al funcionamiento de sistemas cerrados. No obstante, en la segunda generación del pensamiento sistémico, los principios de la teoría general de sistemas de Bertalanffy permitieron emerger al pensamiento agroecológico y sentar las bases para la conceptuación de su unidad de estudio: el agroecosistema. Una de las grandes aportaciones de esta teoría a la agroecología fue el uso de la jerarquía en la definición del objeto de estudio a través de la identificación de subsistemas, sistemas y suprasistemas, facilitando con ello, el diseño metodológico en las investigaciones. Aunque dicho diseño se limitó únicamente al estudio de los principios ecológicos que permitieran sustituir en los agroecosistemas, los insumos externos a través del fomento de procesos naturales como la fertilidad natural del suelo y el control biológico (Altieri 1995).

Bajo este contexto, el estudio de la realidad agrícola utilizando el concepto de agroecosistema, se circunscribió únicamente al estudio de la dimensión ambiental. La influencia de estos principios es evidente en la definición de Hart (1985) quien concibió el agroecosistema como un sistema formado por un grupo de especies en un hábitat específico (factores bióticos) y el medio ambiente (factores abióticos) con el que interactúa procesando entradas y salidas de energía y materiales. En este mismo sentido, Stephen Gliessman (1990) conceptuó al agroecosistema como una unidad de producción agrícola que debe ser comprendida como un ecosistema donde los límites son designados arbitrariamente por el investigador. Desde estos argumentos, el agroecosistema fue considerado un sistema abierto que recibe insumos y genera productos, y su estudio consiste fundamentalmente en el análisis de las relaciones ecológicas: suelo-agua-atmósfera, suelo-planta, planta-insecto, planta-planta, etc.

En otras palabras, la actividad científica agroecológica basada en la segunda generación del pensamiento sistémico se abocó principalmente al estudio de procesos ecológicos con el fin de encontrar un manejo óptimo que indicara cuál debía ser el nivel adecuado de interacciones entre componentes bióticos y abióticos del agroecosistema. Dicho manejo 
requería lograr en el agroecosistema la generación de sinergias que subsidiaran procesos como la activación de la biología del suelo, el reciclado de nutrientes, el aumento de organismos benéficos, entre otros (Altieri \& Nicholls 2000). En estas pretensiones es evidente el predominio del pensamiento cartesiano, al cual subyace el pensamiento del dominium terrae (Luhmann 1996). Un discurso que ha influido en las ciencias y, por ende, en la generación de un acervo importante de conocimientos cuantitativos sobre el funcionamiento de los recursos naturales. No obstante, dicho discurso ha conducido a las tareas de la ciencia descripción, sistemática, explicación y predicción- a un nivel simplemente distributivo que no considera las características del productor y las condiciones específicas de su sitio de producción (Sevilla 2002); con ello se ha ignorado la existencia de un agricultor que realiza su quehacer agrícola en un determinado espacio y tiempo.

En México, Efraín Hernández (1977) fue pionero en introducir el concepto de agroecosistema en el estudio de la realidad agrícola. Concepto que definió como un ecosistema modificado en menor o mayor grado por el hombre al utilizar los recursos naturales en los procesos de producción agrícola. Las aportaciones de este autor, desde su experiencia en diversas regiones del país, abonaron a la conceptualización posterior del agroecosistema (Méndez et al. 2013), es decir, a la valoración del papel de los productores como tomadores de decisiones en las prácticas de manejo del mismo. Así se comenzó a superar en parte, el planteamiento que suponía a la producción agrícola como un proceso objetivo que excluía a los agricultores y su forma de pensar, sus intereses y expectativas (Norgaard \& Sijor 1999). Lo anterior conllevó a que los investigadores consideraran nuevas dimensiones de estudio que permitieran entender, por ejemplo, los flujos que se establecen en y entre los agroecosistemas. Es decir, la conjunción de los intereses entre varios individuos que comparten el quehacer agrícola.

Esta evolución del pensamiento agroecológico, alimentado por las teorías de la segunda generación de pensamiento sistémico, se expresó también en las ideas de Gordon Conway (1987), cuando planteó que los factores más importantes para analizar un agroecosistema son los ambientales, sociales y económicos. Argumento similar al planteado por Octavio Ruiz (1995), al señalar que en el agroecosistema interactuan factores socioeconómicos y tecnológicos para la utilización de los recursos naturales con fines de producción para la obtención de alimento y servicios en beneficio del hombre. Esta idea es coincidente con la propuesta de Miguel Altieri (1995) que reconoció al productor como un componente fundamental de los agroecosistemas, quien es capaz de orientar la producción según sus fines; por lo tanto, el autor planteó que en el estudio de los agroecosistemas se requiere abordar las dimensiones ecológica, social y económica. De este modo, parte de la investigación agroecológica, además de abordar aspectos problemáticos de carácter 
productivo, comenzó a estudiar a los relacionados al comportamiento del mercado. Sin embargo, aunque en estas investigaciones se aludió a los productores como una influencia en la estructura y el funcionamiento de los agroecosistemas, su estudio se dirigió únicamente al control cibernético mínimo ejercido por éstos en los mismos (Elliott \& Vernon 1989; Ruiz-Rosado 2006; Vilaboa-Arroniz et al. 2009; Martínez-Dávila et al. 2011).

Es decir, en la agenda científica agroecológica esto ha conducido a la disociación entre pensamiento sistémico y pensamiento agroecológico. Ya que el primero evolucionó a un nuevo paradigma: el pensamiento complejo, mientras que la mayoría de las investigaciones de carácter agroecológico se sustentan aún en los principios de la teoría general de sistemas y la cibernética de la segunda generación. Un ejemplo de ello son los trabajos desde la unidisciplina que se asumen bajo el enfoque agroecológico con el argumento de profundizar en la investigación dentro de sub-disciplinas académicas específicas (Sevilla 2002; Méndez et al. 2013). Investigaciones que en muchos de los casos se realizan como proyectos de investigación particulares. Esta postura contraviene al discurso desde la agroecología, en cuanto a promover y necesitar de un trabajo multi, inter y transdisciplinar (Ruiz-Rosado 2006; Altieri \& Toledo 2011; Álvarez-Salas et al. 2014).

Es así como la transición del pensamiento agroecológico en concordancia con la emergencia de la tercera generación de pensamiento sistémico ha sido difícil de superar. Un ejemplo es que el agroecosistema aún se sigue conceptuando como algo que existe en la realidad. En otras palabras, esto significa concebir al agroecosistema como un sitio de producción, una unidad de producción equivalente a una granja, finca, parcela, milpa, solar, etc. (Gliessman 2002). Una excepción son Julio Vilaboa-Arroniz (et al. 2009) y Juan P. Martínez-Dávila (et al. 2011), quienes se distinguen por conceptuar al agroecosistema como una construcción lógica, una abstracción que facilitan la interpretación de la realidad agrícola. William Goode y Paul Hatt (2002) mencionan que la tendencia a dar por supuesto que los conceptos existen realmente conduce a la falacia de la objetivación. Esto indica la escasa reflexividad teórica en cuanto a los conceptos, elementos fundamentales del método científico.

Sin embargo, también existen intentos en pro de esa dicha transición, ya que en los últimos años desde el quehacer científico agroecológico se promueve un discurso que pretende conciliar a las ciencias naturales y sociales con el fin de comprender la relación entre los procesos ambientales, económicos y sociales que inciden en la estructura y la función del agroecosistema. En este sentido, Miguel Altieri y Víctor Toledo (2011) propusieron que, en consecuencia, los agrónomos requieren comprender los elementos socioculturales y económicos de los 
agroecosistemas y, a su vez, los científicos sociales se obligan a apreciar los elementos técnicos y ecológicos de éstos. Situación obligada por las condiciones de la agricultura contemporánea, las cuales exigen ir más allá del estudio de una serie de componentes, requiriendo la comprensión del agroecosistema como una totalidad, donde se reconozca al ser humano y su contexto social, un asunto fundamental que influye en la toma de decisiones de lo que se hace o deja de hacer en la actividad agrícola.

Intentos de ello son los planteamientos de Luis Brunett (et al. 2006) que propusieron considerar conocimientos y aspiraciones del productor como factores que influyen en la dinámica del agroecosistema y lo argüido por Octavio Ruiz-Rosado (2006) quien incorpora en su definición de agroecosistema el término "relaciones sociales de producción". En cuanto a Brunett, se evidencia su idea del productor como un individuo fuera de un contexto socio-cultural y socio-político. Mientras que en la definición de Ruiz-Rosado resalta la confusión epistemológica en cuanto al uso indistinto de conceptos asociados a las corrientes de pensamiento materialista e idealista (Mardones \& Ursua 1994), además no profundiza sobre los alcances de estas relaciones sociales en la transformación de la realidad agrícola.

Una aportación interesante es cuando se plantea que el agroecosistema debe ser considerado como un todo que representa mucho más que la simple suma de sus partes y es capaz de tener alta capacidad de resiliencia ante los riesgos de tipo biofísico-ambientales y socioeconómico-políticos a los que están sujetos (Gliessman et al. 2007). Definición que indica el cambio a una conceptuación de agroecosistema como una totalidad y la consideración de comportamientos emergentes como la resistencia y la recuperación del agroecosistema ante una situación contingente. Esta idea es compartida por Raoul Robinson (2007), quien plantea al agroecosistema como un sistema capaz de autoorganizarse. Ambas definiciones expresan el tránsito del pensamiento agroecológico hacia la tercera generación de pensamiento sistémico, la de los sistemas complejos.

Esta apertura a la tercera generación del pensamiento sistémico estaría permitiendo a los investigadores obtener respuestas a cuestionamientos para lo cual antes se encontraban teóricamente limitados. Por ejemplo, ¿cuáles son los límites del agroecosistema? Desde los principios teóricos brindados por la segunda generación de pensamiento sistémico, realizar dicha delimitación presenta dificultades. Si bien se reconoce que las características de los elementos biofísicos influyen en la dinámica de los agroecosistemas, la información de los mercados y las políticas agrícolas también determinan lo que se producirá, cuándo, con qué tecnología, a qué ritmos, para qué clase de consumidores, abriendo más el espectro de lo que puede entenderse como borde o límite de los agroecosistemas (León 2009). Asimismo, ¿cómo deben considerarse los posibles y potenciales impactos 
ambientales de la actividad agrícola? Desde los planteamientos de Herrscher (2005), el límite de un agroecosistema puede separar o unir, depende de su propósito, de la intención de los involucrados, de la eficacia y voluntad de quienes manejan la frontera.

En este punto, se hacen necesarias las aportaciones de la tercera generación de pensamiento sistémico y sus sistemas complejos, modelos teóricos que permitan al investigador el abordaje de la realidad agrícola contemporánea desde la complejidad que les es inherente, permitiendo ir más allá del estudio de los componentes del sistema y abocarse en las interdependencias. Es decir, conceptuando al agroecosistema como un sistema complejo, superando así la idea de que su estudio puede hacerse únicamente a partir de análisis integrados. Esto implicaría que aquellos dedicados a la investigación agroecológica reconozcan y hagan uso de aportaciones teóricas como las de Herrscher (2005), García (2008) y Morin (2007) para de ese modo abordar la influencia de la historia, la cultura y la política en el quehacer agrícola. En consecuencia, la tercera generación de pensamiento sistémico constituye un parteaguas en la evolución del pensamiento agroecológico con teorías consideradas de rango alto con un grado mayor de abstracción, pero cuya ganancia es contar con herramientas cognitivas que permiten la comprensión entre la sociedad y la naturaleza, lo global y lo local, lo espacial y lo temporal. Así, desde estas teorías, la agricultura puede ser estudiada como un sistema complejo con elementos heterogéneos e interdefinibles, siendo condición necesaria el trabajo inter y transdisciplinario (Borssato \& Simões do Carmo 2012; Méndez et al. 2013; Álvarez-Salas et al. 2014; Casanova-Pérez et al. 2015).

No obstante, las aportaciones fundamentales de la tercera generación de pensamiento sistémico para hacer frente a la complejidad agrícola, es importante considerar las aportaciones de la teoría de sistemas sociales autopoiéticos de Luhmann (1989, 2006), cuya arquitectura teórico-conceptual podría considerarse el inicio de una cuarta generación de pensamiento sistémico. Esta teoría se considera útil para el abordaje de problemas como el cambio climático y su impacto en la agricultura, el cual desconoce todo límite político-administrativo, cuyo estudio implica entender el papel de la economía, la política, la ciencia en la evolución de la agricultura contemporánea Sin embargo, la utilización de dicha teoría requiere de la apertura de quienes realizan dicho quehacer científico (agrónomos, ecólogos, biólogos, etc.), ya que es una teoría con un grado mayor de abstracción, pero con la ganancia de contar con herramientas cognitivas que permiten la comprensión entre la sociedad y la naturaleza, lo global y lo local, lo espacial y lo temporal (Álvarez-Salas et al., 2014; Casanova-Pérez et al., 2015).

Desde la teoría de sistemas sociales autopoiéticos, la agricultura se define como un sistema complejo de comunicación que, aunque no tienen límites espaciales definidos suceden en un continuum de realidad 
agrícola. Argumento desde el cual, los productores son ahora parte del entorno, pero con una estrecha relación con el sistema a través de acoplamientos estructurales. En esta arquitectura teórico-conceptual, el agroecosistema es un modelo conceptual que representa a la realidad agrícola, cuyo sistema psíquico (productor) es el receptor de la autopoiesis del sistema agricultura. Autopoiesis que es alimentada por la información que le es notificada a través de medios masivos de comunicación (radio, tv, prensa escrita, internet), medios simbólicamente generalizados (dinero) y por los sistemas de interacción (conversaciones sostenidas entre dos o más productores, productores y técnicos, productores y representantes institucionales, etc.), quienes les proveen de información nueva y valiosa que se usa como referente en la decisiones respecto al manejo de su agroecosistemas. Con el tiempo, esta información seleccionada y actualizada se integrará al conjunto de temas que regularmente se comunican en el contexto local consistente en recuerdos y expectativas que brindan posibilidades al sistema psíquico cuando éste se encuentra ante situaciones inesperadas.

Asimismo, la agricultura es un sistema de comunicación sujeto a repercusiones estructurales debido a su interacción con otros sistemas de su entorno social (política, ciencia, leyes, pero especialmente del sistema economía) y a ajustes estructurales provocados por la información respecto a lo sucedido en su entorno natural. Las repercusiones estructurales suceden cuando entra al sistema agricultura información sobre el comportamiento del sistema de precios de insumos y productos agrícolas, o el descenso o aumento del pago por cosecha por parte de los intermediarios de la producción agrícola. En cuanto a los ajustes estructurales, estos suceden cuando fenómenos naturales generan el suficiente ruido para convertirse en información, generalmente ante la presencia de eventos naturales catastróficos como sequías agudas, los efectos destructivos de un huracán o una inundación, etc. Ambos tipos de información se introducen al sistema de comunicación, utilizando a los medios masivos de comunicación, medios simbólicamente generalizados y la información generada por los sistemas de interacción (cuando se conversa sobre algún tema específico respecto a su quehacer agrícola: bajos precios del grano de maíz, efectos de un estiaje prolongado, etc.).

En consecuencia, toda la información notificada y comprendida por los productores se expresan en el continuum de realidad agrícola en una transformación gradual o abrupta de las prácticas de manejo en los agroecosistemas por parte de los productores (Casanova-Pérez et al. 2015). Expresión de esta transformación es, por ejemplo, la sustitución de maíz nativo por variedades mejoradas de maíz y el uso mayor de agroquímicos. Ambas prácticas forman parte de una estrategia para obtener cosechas cuyos granos cumplan con las expectativas de cantidad y calidad exigidas por el mercado. En contraste, está el abandono de prácticas tradicionales como el uso del trabajo familiar, la ayuda mutua 
entre productores, el trabajo pagado en especie, prácticas basadas en la cooperación y la solidaridad base de la confianza social (Luhmann 1996). Situación que aumenta la vulnerabilidad de los productores ante los efectos del cambio climático y las posibilidades para su adaptación.

Bajo este contexto, se reitera que las teorías que conforman la tercera y la cuarta generación a las cuales subyace el pensamiento sistémico complejo cuentan con los fundamentos necesarios para analizar problemas actuales derivados de fenómenos como el cambio climático y la globalización económica. Los cuales desconocen todo límite políticoadministrativo, cuyo estudio implica entender el papel del poder, la política y la cultura. Sin embargo, la utilización de dichas teorías requiere de la apertura de quienes realizan la investigación agroecológica. Es cierto que estas teorías tienen un grado mayor de abstracción, pero posibilitan al investigador abordar dimensiones o problemas poco explorados. Esto fomentaría y fortalecería los trabajos de carácter inter y transdisciplinarios y con ello, el debate teórico necesario para conducir a la agroecología a su consolidación.

\section{CONCLUSIÓN}

La deconstrucción del concepto agroecosistema fue utilizada como una estrategia para evidenciar la disociación entre la evolución del pensamiento agroecológico y pensamiento sistémico. Este último ha transitado desde hace más de cuatro décadas hacia el pensamiento complejo, con teorías que hoy forman parte de la tercera y la cuarta generación. Teorías cuya arquitectura teórico conceptual proveen de planteamientos radicales para entender a la problemática agrícola contemporánea, pero que han sido ignorados en la actividad científica de carácter agroecológico. Esto como resultado de la escasa reflexividad teórica, del predominio de un enfoque analista y empirista que ha dado origen a análisis integrados que favorecen el estudio de los componentes de los agroecosistemas y no a las interdependencias de los mismos, lo cual impide comprenderlos como una totalidad. Estudios donde el papel del productor como tomador de decisiones en el agroecosistema ha sido expuesto sin considerar sus contextos sociocultural y sociopolítico. Superar lo anterior requiere del pensamiento complejo y sus teorías de rango alto que favorezcan el trabajo de carácter inter y transdisciplinario. Con base en ello se podrá superar la brecha entre el discurso de la agroecología y su actividad científica. $R M$

\section{REFERENCIAS}

Abitbol, P., \& Botero, F. (2005). Teoría de elección racional: estructura conceptual y evolución reciente. Colombia Internacional, 62, 132-145. 
Altieri, M. (1995). El agroecosistema: determinantes, recursos, procesos y sustentabilidad. In M. A. Altieri (Ed.), Agroecología: Bases científicas para una agricultura sustentable (pp. 22-31). Santiago de Chile: CLADES.

Altieri, M. \& Nicholls, C. (2000). Agroecologia: Teoría y práctica para una agricultura sustentable. México DF: Red de Formación Ambiental para América Latina y el Caribe.

Altieri, M. \& Toledo, V. (2011). The Agroecological Revolution of Latin America: Rescuing Nature, Securing Food Sovereignty and Empowering Peasants. Journal of Peasant Studies, 38, 587-612.

Álvarez-Salas, L., Polanco-Echeverry, D., \& Ríos-Osorio, L. (2014). Reflexiones acerca de los aspectos epistemológicos de la agroecología. Cuadernos de Desarrollo Rural, 11(74), 55-74.

Bertalanffy, L. von (1986). Teoría general de sistemas. Fundamentos, desarrollo y aplicaciones. México DF: Fondo de Cultura Económica.

Borsatto, R. \& Simões do Carmo, M. (2012). Agroecologia e sua epistemología. Interciencia, 37(9), 711-716.

Brunett, L., García, L., González, C., De León, F., \& Climent, J. (2006). La agroecología como paradigma para el diseño de la agricultura sustentable y metodologías para su evaluación. Sociedades Rurales, Producción y Medio Ambiente, 6, 84-109.

Calva, J. (2004). Ajuste estructural y TLCAN: efectos en la agricultura mexicana y reflexiones sobre el ALCA. El Cotidiano, 19(14), 14-22.

Cannon, W. (1941). La sabiduría del cuerpo. México DF: Séneca.

Casanova-Pérez, L., Martínez-Dávila, J., López-Ortiz, S., Landeros-Sánchez, C., López Romero, G., \& Peña-Olvera, B. (2015a). Enfoques del pensamiento complejo en el agroecosistema. Interciencia, 40(3), 210-217.

Checkland, P. (1993). Pensamiento de sistemas, práctica de sistemas. México DF: Noriega Editores.

Chiavenato, I. (1997). Introducción a la teoría general de la administración. Bogotá: McGraw Hill.

Conway, G. (1987). The Properties of Agroecosystems. Agricultural Systems, 24, 95117.

Durán, C. (2013). Teoría de sistemas y deconstrucción. Cinta de Moebio, 84, 26-36.

Elliott, E. \& Vernon C. (1989). A Perspective on Agroecosystem Science. Ecology, 70, 1597-1602.

Forrester, J. (1989). The Beginning of System Dynamics. Banquet Talk at the International Meeting of the System Dynamics Society, July 13. Stuttgart, Germany. Consultado el 21 de octubre, 2014, desde http://web.mit.edu/sysdyn/sdintro/D-4165-1.pdf.

García, R. (2008). Sistemas complejos. Barcelona: Gedisa.

Gharajedagui, J. (2008). Prólogo. En E. Herrscher (Autor), Pensamiento sistémico, caminar el cambio o cambiar el camino (pp. 11-23). Buenos Aires: Granica.

Gliessman, S. (1990). Researching the Ecological Basis for Sustainable Agriculture. Agroecology, 78, 3-10

Gliessman, S. (2002). Agroecología: Procesos ecológicos en agricultura sostenible. Turrialba: Centro Agronómico Tropical de Investigación y Enseñanza.

Gliessman, S. (2005). Sin la agroecología no se puede concebir un desarrollo sostenible. Sustrai, 71, 4-9.

Gliessman, S., Rosado-May, F., Guadarrama-Zugasti, C., Jedlicka, J., Cohn, A., Méndez, V., Cohen, R., Trujillo, L., Bacon, C., \& Jaffe, R. (2007). Agroecología: promoviendo una transición hacia la sostenibilidad. Ecosistemas, 16(1), 13-23.

Goode, W., \& Hatt, P. (2002). Métodos de investigación social. México DF: Trillas. 
Hart, R. (1985). Conceptos básicos sobre agroecosistemas. Turrialba: Centro Agronómico Tropical de Investigación y Enseñanza.

Hernández, E. (1977). El agroecosistema: Concepto central en el análisis de la enseñanza, la investigación y la educación agrícola en México. En E. Hernández (Ed.), Agroecosistemas de México: contribuciones a la enseñanza, investigación y divulgación agrícola (pp. XI-XIX) Chapingo Méx.: Colegio de Postgraduados.

Herrscher, E. (2005). Pensamiento sistémico, caminar el cambio o cambiar el camino. Buenos Aires: Granica.

Krieger, P. (2004). La deconstrucción de Jacques Derrida. Anales del Instituto de Investigaciones Estéticas, 84, 179-188.

León, T. (2009). Agroecología: Desafíos de una ciencia ambiental en construcción. En M. A. Altieri (Ed). Vertientes del pensamiento agroecológico: fundamentos y aplicaciones. (pp: 46-67) Medellín: SOCLA.

Lilienfeld, R. (1984). Teoría de sistemas. Orígenes y aplicaciones en ciencias sociales. México DF: Trillas.

Luhmann, N. (1989). Ecological Communication. Chicago: University of Chicago Press.

Luhmann, N. (1996). Introducción a la teoría de sistemas. México DF: Universidad Iberoamericana - Instituto Tecnológico de Occidente.

Luhmann, N. (2006). La sociedad de la sociedad. México DF: Herder, Universidad Iberoamericana.

Mardones, J. \& Ursua, N. (1994). Filosofía de las ciencias humanas y sociales. Materiales para una fundamentación científica. Barcelona: Fontanamara.

Martínez, J., Gallardo, F., Bustillo, L., \& Pérez, A. (2011). El agroecosistema, unidad de estudio y transformación de la diversidad agrícola en Veracruz. En A. Cruz (Ed.), La biodiversidad en Veracruz. Volumen I. Xalapa: Universidad VeracruzanaInstituto de Ecología A.C.

Méndez, E., Bacon, C., \& Cohen, R. (2013). La agroecología como un enfoque transdisciplinario, participativo y orientado a la acción. Agroecología, 8, 9-18.

Morin, E. (2007). Introducción al pensamiento complejo. Barcelona: Gedisa.

Norgaard, R. \& T. Sikor. 1999. Metodología y práctica de la agroecología. En M.A. Altieri (Ed.), Agroecología. Bases científicas para una agricultura sustentable (pp. 27-42). Montevideo: Editorial Nordan-Comunidad.

Neumann, J. (1948). The General and Logical Theory of Automata. En A. Taub (Ed.), Design of computers, theory of automata and numerical analysis (pp: 289-396). Urbana-Champaign: Pergamon Press.

O'Brien, K. \& Leichenko, R. (2000). Double Exposure: Assessing the Impacts of Climate Change within the Context of Economic Globalization. Global Environmental Change, 10, 221-232.

Robinson, R. (2007). Self-organising Agroecosystems. Canada: Sharebooks Publishing. Ruiz, O. (1995). Agroecosistema. Término, concepto y su definición bajo el enfoque agroecológico y sistémico. En Memoria del Seminario Internacional de Agroecología. Chapingo, Méx.: UACH.

Ruiz-Rosado, O. (2006). Agroecología: una disciplina que tiende a la transdisciplina. Interciencia, 31(2), 140-145.

Rivera, C. (2009). La agroecología: cruce de fronteras epistemológicas. Memoria del XXVII Congreso de la Asociación Latinoamericana de Sociología. Asociación Latinoamericana de Sociología. Buenos Aires.

Sáez, F. (2009). Complejidad y tecnologías de la información. Madrid: Fundación Rogelio Segovia para el Desarrollo de las Telecomunicaciones. 
Sevilla, E. (2002). La agroecología como estrategia metodológica de transformación social. Instituto de Sociología y Estudios Campesinos de la Universidad de Córdoba. España.

Vilaboa-Arroniz, J., Díaz-Rivera, P., Ruiz-Rosado, O., Platas-Rosado, D., GonzálezMuñoz, S., \& Juárez-Lagunes, F. (2009). Caracterización socioeconómica y tecnológica de los agroecosistemas con bovinos de doble propósito de la región del Papaloapan, Veracruz, México. Tropical and Subtropical Agroecosystems, 10, 53-62.

Van Gigch, J. P. (1990). Teoría general de sistemas. México DF: Trillas.

Weaver, W. (1964). Recent Contributions to the Mathematical Theory of Communication. In C. Shannon \& W. Weaver (Autores), The Mathematical Theory of Communication. (pp: 1-28) Urbana: University of Illinois Press.

Weaver, W. (2004). Science and complexity. Classical Papers, 6(3), 65-74.

SOBRE LOS AUTORES

Lorena Casanova-Pérez es Ingeniero Agrónomo con la especialidad en Economía por la Universidad Autónoma Chapingo, México. Maestra en Ciencias en Recursos Naturales y Desarrollo Rural por el Colegio de la Frontera Sur, México. Doctora en Ciencias en Agroecosistemas Tropicales por El Colegio de Postgraduados, México. casanova.lorena@colpos.mx

Juan Pablo Martínez-Dávila es Ingeniero Civil por la Universidad Autónoma de Veracruz, México. Maestro en Ciencias en Estrategias para el Desarrollo Agrícola Regional. Colegio de Postgraduados, México. Doctor en Ciencias en Agroecosistemas Tropicales, Colegio de Postgraduados, México.

jpmartin@colpos.mx

Silvia López-Ortiz es Ingeniero Agrónomo Zootecnista por el Instituto Tecnológico Agropecuario Úrsulo Galván, México. Maestra en Ciencias Agropecuarias y de los Recursos Naturales, CATIE, Costa Rica y Doctor of Philosophy por la Universidad de Idaho, USA.

silvialopez@colpos.mx

Gustavo López Romero es Ingeniero Agrónomo por la Universidad Autónoma Chapingo, México. Maestro en Ciencias en Genética por el Colegio de Postgraduados, México. Doctor en Ciencias en Genética por el Colegio de Postgraduados, México. gustavolr@colpos.mx

\section{CONTACTO}

Colegio de Postgraduados, Campus Veracruz,

Programa Agroecosistemas Tropicales

Km 85.5, Carretera Xalapa-Veracruz, Predio Tepetates, municipio de Manlio Fabio Altamirano,

Veracruz, México C.P. 91690,

Apartado postal 91700

casanova.lorena@colpos.mx

Recibido: abril 2016

Aceptado: julio 2016 